Anaesthesist 2009 - 58:247-258 DOI 10.1007/s00101-008-1485-9 Online publiziert: 2. März 2009

(c) Springer Medizin Verlag 2009

G. Erdös ${ }^{1} \cdot$ I. Tzanova ${ }^{2} \cdot$ U. Schirmer ${ }^{3} \cdot$ J. Ender ${ }^{4}$

${ }^{1}$ Inselspital, Universitätsklinik für Anästhesiologie und Schmerztherapie, Bern

${ }^{2}$ Klinik für Anästhesiologie, Universitätsmedizin der Johannes-Gutenberg-Universität Mainz

${ }^{3}$ Abteilung Kardioanästhesiologie, Universitätsklinikum Ulm

${ }^{4}$ Abteilung für Anästhesie und Intensivmedizin II, Herzzentrum, Universität Leipzig

\title{
Neuromonitoring und Neuroprotektion in der Kardioanästhesie
}

\section{Bundesweite Umfrage des Arbeitskreises Kardioanästhesie der Deutschen Gesellschaft für Anästhesiologie und Intensivmedizin e.V.}

Der kardiochirurgische Patient weist gegenüber Patientengruppen anderer operativer Fächer ein deutlich höheres Risiko perioperativer neurologischer Komplikationen auf [45]. So schätzt Guarracino [24] die Inzidenz von Apoplex nach Herzoperationen auf bis zu $6 \%$. Andere Störungen, wie die durch normierte Gedächtnisund Lerntests qualifizierbare Beeinträchtigung der kognitiven Funktion treten sogar bei bis zu $60 \%$ der Patienten in der ersten postoperativen Woche auf und sind in $\mathbf{4 5 \%}$ der Fälle bis zu 5 Jahre nach der Operation nachweisbar [31]. Hieraus ergibt sich der Bedarf an effektiven Maßnahmen des Neuromonitorings und der Neuroprotektion in der Kardioanästhesie. Mithilfe der im Folgenden beschriebenen Umfrage sollte herausgefunden werden, ob und inwiefern diesem Bedarf Genüge getan wird.

Postoperative neurologische und neuropsychologische Defizite

Die sozioökonomischen Auswirkungen postoperativer neurologischer und neuropsychologischer Defizite (PNCD) sind erheblich. Neben der psychischen Belastung für den Patienten selbst und dessen soziales Umfeld sind sie mit erheblichen Kosten im Gesundheitswesen verbunden. Nach einer Untersuchung von Mangano aus dem Jahr 1994 betrugen in den USA bis dato die jährlichen Zusatzkosten für verlängerten Krankenhausaufenthalt bzw. für Posthospitalversorgung und Rehabilitation nach einem PNCD als Folge eines Eingriffs mit extrakorporaler Zirkulation 750 Mio. bzw. 500 Mio. US-Dollar [37].

Die vollständige Vermeidung von PN$\mathrm{CD}$ in der Kardiochirurgie ist derzeit nicht möglich. Mit entsprechendem technischen Aufwand, alternativen chirurgischen Verfahren und durch Ausbildung bzw. Sensibilisierung des medizinischen Personals lässt sich jedoch die Inzidenz signifikant reduzieren [15].

\section{Verfahren zur Überwachung der Hirnfunktion}

In der heutigen kardioanästhesiologischen Praxis existiert eine Vielzahl verschiedener Verfahren zur Überwachung der Hirnfunktion. Während mit älteren Techniken wie Elektroenzephalographie
(EEG) und Ableitung evozierter Potenziale (EP) die Detektion der elektrischen Hirnaktivität möglich ist, können moderne Verfahren, wie die transkranielle Dopplersonographie (TCD) und die Nahinfrarotspektroskopie (NIRS) Informationen über Hirndurchblutung und -metabolismus liefern. Jede dieser Methoden verfügt über spezifische Vor- sowie Nachteile und erlaubt, das zentrale Nervensystem (ZNS) aus einem anderen Blickwinkel zu betrachten. Ein einzelnes optimales Verfahren gibt es nicht. Je nach Verfügbarkeit und persönlicher Präferenz werden unterschiedliche zerebrale Überwachungsmethoden eingesetzt.

\section{Bundesweite Umfrage}

Ziele

Ziel der vorliegenden Studie war es, einen deutschlandweiten Überblick über die aktuelle Praxis des Neuromonitorings in der Kardioanästhesie zu gewinnen. Das Interesse galt hauptsächlich dem intraoperativen Einsatz von Neuromonitoringverfahren und der Anwendung neuroprotektiver Maßnahmen. Als Sekundäraspekte wurden folgende Parameter erfasst: 


\section{Präoperative Evaluation}

a) Erfolgt eine regelmässige präoperative Diagnostik der hirnversorgenden Arterien?

$\square$ Immer

$\square$ Nur bei klinischer Symptomatik

$\square$ Nur vor entsprechender Operation (ACI-TEA in Kombination mit Herzchirurgie)

$\square$ Selten

$\square$ Nie

b) Die Untersuchung erfolgt mittels

$\square$ Duplex-Sonographie

$\square$ Transkranieller Dopplersonographie (TCD)

$\square$ Angiographie

$\square$ Cerebraler Computertomographie (CCT) bzw. Angio-CCT (CCT-A)

$\square$ Magnetresonanztomographie (MRT)

c) Wer ordnet die entsprechende Untersuchung an?

$\square$ Der Anästhesist

$\square$ Der Operateur

$\square$ Der Stationsarzt

$\square$ Der Neurologe

d) Wer legt das weitere therapeutische Procedere auf Grundlage des zuvor erhobenen Befundes fest?

$\square$ Der Anästhesist

$\square$ Der Operateur

$\square$ Der Stationsarzt

$\square$ Der Neurologe

e) Wer wählt das adäquate Neuromonitoring-Verfahren aus?

$\square$ Der Anästhesist

$\square$ Der Operateur

$\square$ Der Stationsarzt

$\square$ Der Neurologe

\section{Neuromonitoring-Verfahren}

a) Welche Neuromonitoring-Verfahren sind in Ihrer Abteilung verfügbar?

$\square$ Elektroenzephalogramm (EEG)

$\square$ Evozierte Potentiale (EP) (z. B. Medianus-, Tibialis-SSEP, MEP, AEP)

$\square$ Infrarotnahe Spektroskopie (NIRS)

$\square$ Transkranielle Dopplersonographie (TCD)

$\square$ Jugularvenöse Sauerstoffsättigung $\left(\mathrm{S}_{\mathrm{v}} \mathrm{O}_{2}\right)$

$\square$ Stumpfdruckmessung der A. carotis interna (SDM)

$\square$ Postoperative Bestimmung von Markern (NSE, GAP)

$\square$ Keine ( $>$ bitte bei Frage 3 weitermachen)

b) Welche Neuromonitoring-Verfahren werden genutzt?

$\square$ Elektroenzephalogramm (EEG)

$\square$ Evozierte Potentiale (EP) (z. B. Medianus-, Tibialis-SSEP, MEP, AEP)

$\square$ Infrarotnahe Spektroskopie (NIRS)

$\square$ Transkranielle Dopplersonographie (TCD)

$\square$ Jugularvenöse Sauerstoffsättigung $\left(\mathrm{S}_{\mathrm{y}} \mathrm{O}_{2}\right)$

$\square$ Stumpfdruckmessung der A. carotis interna (SDM)

$\square$ Postoperative Bestimmung von Markern (NSE, GAP)

$\square$ Keine ( $>$ bitte bei Frage 3 weitermachen)

c) Wie häufig wird das entsprechende Neuromonitoring-Verfahren genutzt?

$\square$ Immer, wenn von Seiten des Patienten oder der Operation indiziert

$\square$ Nur bei speziellen Fragestellungen

$\square$ Selten

$\square$ Nie ( $>$ bitte bei Frage 3 weitermachen)

d) Wer schliesst die Anlage intraoperativ an?

$\square$ Der Anästhesist

$\square$ Eine anästhesiologische Pflegekraft

$\square$ Der Chirurg bzw. chirurgischer Assistent

$\square$ Eine speziell für Neuromonitoring ausgebildete Fachkraft

e) Wer bewertet intraoperativ die Ergebnisse?

$\square$ Der Anästhesist

$\square$ Eine anästhesiologische Pflegekraft

$\square$ Der Chirurg bzw. chirurgischer Assistent

$\square$ Eine speziell für Neuromonitoring ausgebildete Fachkraft f) Wann werden die Ergebnisse ausgewertet?

$\square$ Bereits intraoperativ (online)

$\square$ Direkt postoperativ

Postoperativ, bis zum 1. postoperativen Tag

$\square$ Postoperativ, nach dem 1. postoperativen Tag

g) Welches Neuromonitoring-Verfahren benutzen Sie bei

$\triangle$ Typ-A-Dissektion

$\square$ EEG $\quad \square$ EP $\quad \square$ NIRS $\quad \square$ TCD $\square \mathrm{S}_{\mathrm{yi}} \mathrm{O}_{2} \quad \square$ Marker $\quad \square$ Keine

$\triangle$ Aortenchirurgie

$\square$ EEG $\square$ EP $\square$ NIRS $\square$ TCD $\square \mathrm{S}_{\mathrm{yj}} \mathrm{O}_{2} \quad \square$ Marker $\square$ Keine

$\triangle$ Thorakoabdominellem Aortenersatz

$\square$ EEG $\quad \square$ EP $\quad \square$ NIRS $\quad \square$ TCD $\square \mathrm{S}_{\mathrm{vi}} \mathrm{O}_{2} \quad \square$ Marker $\square$ Keine

$\triangle$ Karotischirurgie in Kombination mit Herzchirurgie

$\square$ EEG $\square$ EP $\square$ NIRS $\square$ TCD $\square \mathrm{S}_{\mathrm{vi}} \mathrm{O}_{2} \quad \square$ Marker $\square$ Keine

$\square$ Stumpdruckmessung der A. carotis interna (SDM)

$\triangle A C B$

$\square$ EEG $\quad \square$ EP $\quad \square$ NIRS $\quad \square$ TCD $\square S_{\text {vij }} \mathrm{O}_{2} \quad \square$ Marker $\quad \square$ Keine

$\triangle$ Beating heart-ACB (OPCAB)

$\square$ EEG $\square$ EP $\square$ NIRS $\square$ TCD $\square \mathrm{S}_{\mathrm{vj}} \mathrm{O}_{2} \quad \square$ Marker $\square$ Keine

$\triangleright$ Aortenklappenchirurgie

$\square$ EEG $\quad \square$ EP $\quad \square$ NIRS $\quad \square$ TCD $\quad \square \mathrm{S}_{\mathrm{vi}} \mathrm{O}_{2} \quad \square$ Marker $\quad \square$ Keine

$\triangle$ Chirurgie der übrigen Klappen

$\square$ EEG $\square$ EP $\square$ NIRS $\square$ TCD $\square S_{\mathrm{vj}} \mathrm{O}_{2} \quad \square$ Marker $\square$ Keine

$\triangle$ Minimal-invasiver Aortenklappenchirurgie

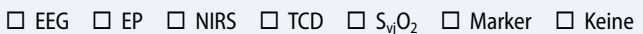

$\triangle$ Minimal-invasiver Mitralklappenchirurgie

$\square$ EEG $\square$ EP $\square$ NIRS $\square$ TCD $\square S_{\text {vi }} \mathrm{O}_{2} \quad \square$ Marker $\square$ Keine

$\triangle$ Minimal-invasiver Bypasschirurgie (MIDCAB)

$\square$ EEG $\square$ EP $\square$ NIRS $\square$ TCD $\square$ Svj $_{\mathrm{O}_{2}} \quad \square$ Marker $\square$ Keine

$\triangle$ Chirurgie von angeborenen/erworbenen Vitien

$\square$ EEG $\quad \square$ EP $\quad \square$ NIRS $\quad \square$ TCD $\quad \square S_{\text {vij }} \mathrm{O}_{2} \quad \square$ Marker $\square$ Keine

\section{Cerebroprotektion}

a) Welche cerebroprotektive Massnahmen halten Sie bei Operationen mit Herz-Kreislauf-Stillstand für indiziert?

Medikamentöse Verfahren

$\square$ Kortikosteroide

$\square$ Barbiturate

$\square$ Antiepileptika

$\square$ Externe Kühlung des Kopfes (Ice-CAP)

$\square$ Kühlung an der Herz-Lungenmaschine (HLM)

$\square$ Keine

$\square$ Alle

b) Bei welchen Operationen erfolgt eine Kühlung des Patienten?

$\square$ Bei allen Operationen mit Herz-Lungenmaschine (HLM)

Bei Operationen mit extrakorporaler Zirkulation > 2 Std

$\square$ Bei Operationen an den Herzklappen

$\square$ Bei Operationen an den Koronargefässen

$\square$ Bei Operationen an den herznahen Gefässen

$\square$ Bei Operationen mit Herz-Kreislauf-Stillstand

$\square$ Eine Entscheidung darüber wird individuell (Operateur) getroffen

c) Auf maximal welche Körperkerntemperatur werden die Patienten gekühlt?

$\triangleright$ Typ-A-Dissektion

$\square 34-32^{\circ} \mathrm{C} \quad \square 31-28^{\circ} \mathrm{C} \quad \square 27-24^{\circ} \mathrm{C} \quad \square 23-18^{\circ} \mathrm{C} \quad \square<18^{\circ} \mathrm{C}$

$\triangleright$ Aortenchirurgie

$\square 34-32^{\circ} \mathrm{C} \quad \square 31-28^{\circ} \mathrm{C} \quad \square 27-24^{\circ} \mathrm{C} \quad \square 23-18^{\circ} \mathrm{C} \quad \square<18^{\circ} \mathrm{C}$

$\triangleright$ Thorakoabdomineller Aortenersatz

$\square 34-32{ }^{\circ} \mathrm{C} \quad \square 31-28^{\circ} \mathrm{C} \quad \square 27-24^{\circ} \mathrm{C} \quad \square 23-18^{\circ} \mathrm{C} \quad \square<18^{\circ} \mathrm{C}$

$\triangleright$ Karotischirurgie in Kombination mit Herzchirurgie

$\square 34-32^{\circ} \mathrm{C} \quad \square 31-28^{\circ} \mathrm{C} \quad \square 27-24^{\circ} \mathrm{C} \quad \square 23-18^{\circ} \mathrm{C} \quad \square<18^{\circ} \mathrm{C}$ $\triangle A C B$

$\square 34-32^{\circ} \mathrm{C} \quad \square 31-28^{\circ} \mathrm{C} \quad \square 27-24^{\circ} \mathrm{C} \quad \square 23-18^{\circ} \mathrm{C} \quad \square<18{ }^{\circ} \mathrm{C}$

Abb. $1 \Delta$ Fragebogen: Neuromonitoring und -protektion in der Kardioanästhesie deutscher Kliniken 


\section{G. Erdös $\cdot$ I. Tzanova $\cdot$ U. Schirmer $\cdot$ J. Ender}

Neuromonitoring und Neuroprotektion in der Kardioanästhesie. Bundesweite Umfrage des Arbeitskreises Kardioanästhesie der Deutschen Gesellschaft für Anästhesiologie und Intensivmedizin e.V.

\section{Zusammenfassung}

Fragestellung. Primäres Ziel dieser in deutschen kardioanästhesiologischen Abteilungen durchgeführten Umfrage war, die aktuelle Praxis von Neuromonitoring und Neuroprotektion zu erheben.

Methodik. Zwischen Oktober 2007 und Januar 2008 wurden hierzu Daten mithilfe eines 26 Punkte umfassenden, anonymisierten Fragebogens erhoben. Ermittelt wurden: präoperative Evaluation der hirnversorgenden Arterien, intraoperatives Neuromonitoring, zerebroprotektive Maßnahmen, Perfusionsmanagement während extrakorporaler Zirkulation, postoperative Erhebung des neurologischen Status und Aus-/Weiterbildung im zerebralen Monitoring.

Ergebnisse. 55\% der Fragebögen wurden mit folgenden Angaben beantwortet: präoperative Duplexsonographie der Hirngefäße in $90 \%$ der Kliniken; intraoperatives Neuromonitoring mithilfe der Elektroenzephalographie (EEG; 60\%) bei Typ-A-Dissektionen (38,1\%), bei elektiven Operationen an der thorakalen bzw. thorakoabdominellen Aorta (34,1\% resp. $31,6 \%$ ) und in der Karotischirurgie (43,2\%), weiterhin Einsatz der Nahinfrarotspektroskopie (40\%), Ableitung evozierter Potenziale (30\%) und transkranielle Dopplersonographie (17,5\%). Auch kombinierte Verfahren wurden angewandt. Während Bypass-, Klappen und minimal-invasiven Operationen erfolgt meistens kein Monitoring des Zentralnervensystems. Zur Zerebroprotektion werden die Kühlung des Patienten an Herz-Lungen-Maschine (HLM; 100\%), externe Kühlung des Kopfes (65\%), Gabe von Kortikosteroiden (58\%), Barbituraten (50\%) und Antiepileptika (10\%) eingesetzt. Als neuroprotektive Anästhesieverfahren gelten Inhalationsanästhesie (32,5\%; Favorit: Sevofluran $76,5 \%)$ und total intravenöse Anästhesie (20\%; Favoriten: Propofol und Barbiturate mit je 46,2\%). Standardmäßig kühlen $72,5 \%$ der Krankenhäuser die Patienten bei Operationen mit Herz-Kreislauf-Stillstand, $37,5 \%$ bei allen Operationen mit HLM. Unter normothermen Bedingungen entspricht in $84,6 \%$ der Kliniken der HLM-Fluss dem errechneten Herzzeitvolumen (HZV), der anzustrebende mittlere arterielle Druck (MAP) liegt bei $60-70 \mathrm{mmHg}(43,9 \%)$ bzw. 50-60 mm-
$\mathrm{Hg}$ (41,5\%). Bei einer Körpertemperatur unter $18^{\circ} \mathrm{C}$ wird der HLM-Fluss unter das errechnete HZV gesenkt (70\%), während in $27 \%$ der Kliniken normotherme Flussraten gefahren werden. Der bevorzugte MAP unter Hypothermie liegt zwischen 50 und $60 \mathrm{mmHg}$ (59\%). Intraoperatives Neuromonitoring wird im Narkoseprotokoll (77\%) dokumentiert. Postoperativ wird der neurologische Status in 42,5\% der Kliniken durch individuelle Einschätzung des Anästhesisten $(77,5 \%)$ erhoben. Fortbildungen zum Thema Neuromonitoring werden in $32,5 \%$ der Kliniken regelmäßig organisiert, in $37,5 \%$ dem Arzt selbst überlassen.

Schlussfolgerung. Das kardioanästhesiologische Vorgehen in Deutschland ist im Bereich Neuromonitoring und neuroprotektive Therapie nicht standardisiert. Ein "multimodales Neuromonitoring" wäre wünschenswert.

Schlüsselwörter Neuromonitoring · Neuroprotektion · Perfusionsmanagement $\cdot$ Kardioanästhesie $\cdot$ Neurologisches Defizit

\section{Neuromonitoring and neuroprotection in cardiac anaesthesia. Nationwide survey conducted by the Cardiac Anaesthesia Working Group of the German Society of Anaesthesiology and Intensive Care Medicine}

\section{Abstract}

Objective. The primary objective of this nationwide survey carried out in department of cardiac anesthesia in Germany was to identify current practice with regard to neuromonitoring und neuroprotection.

Methodology. The data are based on a questionnaire sent out to all departments of cardiac anesthesia in Germany between October 2007 und January 2008. The anonymized questionnaire contained 26 questions about the practice of preoperative evaluation of cerebral vessels, intra-operative use of neuromonitoring, the nature und application of cerebral protective measures, perfusion management during cardiopulmonary bypass, postoperative evaluation of neurological status, and training in the field of cerebral monitoring.

Results. Of the 80 mailed questionnaires $55 \%$ were returned and $90 \%$ of department evaluated cerebral vessels preoperatively with duplex ultrasound. The methods used for intra-operative neuromonitoring are electroencephalography (EEG, $60 \%$ ) for type A dissections (38.1\%), for elective surgery on the thoracic and thoraco-abdominal aorta $34.1 \%$ and $31.6 \%$, respectively) and in carotid sur- gery (43.2\%) near infrared spectroscopy (40\%), evoked potentials (30\%) and transcranial Doppler sonography (17.5\%), with some centers using combined methods. In most departments the central nervous system is not subjected to monitoring during bypass surgery, heart valve surgery, or minimally invasive surgery. Cerebral protective measures used comprise patient cooling on cardio-pulmonary bypass (CPB 100\%), extracorporeal cooling of the head (65\%) and the administration of corticosteroids (58\%), barbiturates (50\%) and antiepileptic drugs (10\%). Neuroprotective anesthesia consists of administering inhalation anesthetics (32.5\%; sevoflurane $76.5 \%$ ) and intravenous anesthesia (20\%; propofol and barbiturates each accounting for $46.2 \%)$. Of the departments $72.5 \%$ cool patients as a standard procedure for surgery involving cardiovascular arrest and $37.5 \%$ during all surgery using CPB. In $84.6 \%$ of department CPB flow equals calculated cardiac output (CO) under normothermia, while the desired mean arterial pressure (MAP) varies between 60 and $70 \mathrm{mmHg}$ (43.9\%) and between 50 and $60 \mathrm{mmHg}$ (41.5\%), respectively. At body temperatures less than $18^{\circ} \mathrm{C} C P B$ flow is reduced below the calculated CO (70\%) while $27 \%$ of departments use normothermic flow rates. The preferred MAP under hypothermia is between 50 and $60 \mathrm{mmHg}(59 \%)$. The results of intra-operative neuromonitoring are documented on the anesthesia record (77\%). In $42.5 \%$ of the departments postoperative neurological function is estimated by the anesthesiologist. Continuing education sessions pertaining to neuromonitoring are organized on a regular basis in $32.5 \%$ of the departments and in $37.5 \%$ individual physicians are responsible for their own neuromonitoring education.

Conclusion. The present survey data indicate that neuromonitoring and neuroprotective therapy during CPB is not standardized in cardiac anesthesiology departments in Germany. The systemic use of available methods to implement multimodal neuromonitoring would be desirable.

\section{Keywords}

Neuromonitoring · Neuroprotection · Perfusion management · Cardiac anaesthesia · Neurologic deficit 
$\triangle$ Beating heart- $\mathrm{ACB}$

$\square 34-32^{\circ} \mathrm{C} \quad \square 31-28^{\circ} \mathrm{C} \quad \square 27-24^{\circ} \mathrm{C} \quad \square 23-18^{\circ} \mathrm{C} \quad \square<18^{\circ} \mathrm{C}$

$\triangleright$ Aortenklappenchirurgie

$\square 34-32^{\circ} \mathrm{C} \quad \square 31-28^{\circ} \mathrm{C} \quad \square 27-24^{\circ} \mathrm{C} \quad \square 23-18^{\circ} \mathrm{C} \quad \square<18^{\circ} \mathrm{C}$

$\triangleright$ Chirurgie der übrigen Klappen

$\square 34-32{ }^{\circ} \mathrm{C} \quad \square 31-28^{\circ} \mathrm{C} \quad \square 27-24^{\circ} \mathrm{C} \quad \square 23-18^{\circ} \mathrm{C} \quad \square<18{ }^{\circ} \mathrm{C}$

$\triangleright$ Minimal-invasive Aortenklappenchirurgie

$\square 34-32^{\circ} \mathrm{C} \quad \square 31-28^{\circ} \mathrm{C} \quad \square 27-24^{\circ} \mathrm{C} \quad \square 23-18^{\circ} \mathrm{C} \quad \square<18^{\circ} \mathrm{C}$

$\triangleright$ Minimal-invasive Mitralklappenchirurgie

$\square 34-32{ }^{\circ} \mathrm{C} \quad \square 31-28^{\circ} \mathrm{C} \quad \square 27-24^{\circ} \mathrm{C} \quad \square 23-18^{\circ} \mathrm{C} \quad \square<18^{\circ} \mathrm{C}$

$\triangleright$ Chirurgie von angeborenen/erworbenen Vitien

$$
\square 34-32^{\circ} \mathrm{C} \quad \square 31-28^{\circ} \mathrm{C} \quad \square 27-24^{\circ} \mathrm{C} \quad \square 23-18^{\circ} \mathrm{C} \quad \square<18{ }^{\circ} \mathrm{C}
$$

d) Gibt es eine besondere Kombination von Narkosemedikamenten,

um neuroprotektive Anästhesie durchzuführen?

$\triangleright$ Betonung der Inhalationsanästhesie

$\square$ Sevofluran

$\square$ Desfluran

$\square$ Andere

$\triangleright$ Betonung der Injektionsanästhesie

$\square$ Propofol

$\square$ Barbiturate

$\square$ Benzodiazepine

$\square$ Opiatbetonte Narkoseführung

$\square$ Keine besondere Medikamentenwahl notwendig

\section{Management der extrakorporalen Zirkulation}

a) Welche HLM-Flussraten werden zur ausreichenden cerebralen Perfusion bei Normothermie genutzt?

$\square$ HLM-Flow = errechnetes Herz-Zeit-Volumen (HZV) $\left(=2-2,6 \mathrm{l} / \mathrm{min} / \mathrm{m}^{2}\right)$

$\square$ HLM-Flow grösser als errechnetes HZV HLM-Flow: ............

$\square$ HLM-Flow kleiner als errechnetes HZV HLM-Flow:

b) Welchen mittleren arteriellen Druck (MAP) streben Sie zur ausreichenden cerebralen Perfusion bei Normothermie an?

$\square$ MAP grösser als $70 \mathrm{mmHg}$

$\square$ MAP zwischen $70-60 \mathrm{mmHg}$

$\square$ MAP zwischen $60-50 \mathrm{mmHg}$

$\square$ MAP kleiner als $50 \mathrm{mmHg}$

$\square$ Keinen bestimmten MAP

c) Welche HLM-Flussraten werden zur ausreichenden cerebralen Perfusion bei Hypothermie genutzt?

$\triangleright$ Bei 34 - 28 Grad Körperkerntemperatur?

$\square$ HLM-Flow = errechnetes Herz-Zeit-Volumen $(\mathrm{HZV})\left(=2-2,6 \mathrm{l} / \mathrm{min} / \mathrm{m}^{2}\right)$

$\square$ HLM-Flow grösser als errechnetes HZV HLM-Flow: ............

$\square$ HLM-Flow kleiner als errechnetes HZV HLM-Flow: ...........

$\triangle$ Bei unter 28 Grad Körperkerntemperatur?

$\square$ HLM-Flow = errechnetes Herz-Zeit-Volumen $(\mathrm{HZV})\left(=2-2,6 \mathrm{l} / \mathrm{min} / \mathrm{m}^{2}\right)$

$\square$ HLM-Flow grösser als errechnetes HZV HLM-Flow: ............

$\square$ HLM-Flow kleiner als errechnetes HZV HLM-Flow: ............

$\triangleright$ Bei unter 18 Grad Körperkerntemperatur?

$\square$ HLM-Flow = errechnetes Herz-Zeit-Volumen $(\mathrm{HZV})\left(=2-2,6 \mathrm{l} / \mathrm{min} / \mathrm{m}^{2}\right)$

$\square$ HLM-Flow grösser als errechnetes HZV HLM-Flow:

$\square$ HLM-Flow kleiner als errechnetes HZV HLM-Flow: ............

d) Welchen MAP streben Sie zur ausreichenden cerebralen Perfusion bei Hypothermie an?

$\square$ MAP grösser als $70 \mathrm{mmHg}$

$\square$ MAP zwischen $70-60 \mathrm{mmHg}$

$\square$ MAP zwischen $60-50 \mathrm{mmHg}$

$\square$ MAP kleiner als $50 \mathrm{mmHg}$

$\square$ Keinen bestimmten MAP

\section{Dokumentation}

a) Werden die Ergebnisse / Befunde des cerebralen Neuromonitorings dokumentiert oder gespeichert?

$\square$ Dokumentation und Speicherung auf Narkoseprotokoll

$\square$ Dokumentation und Speicherung separat auf Papier

$\square$ Dokumentation und Speicherung separat in elektronischer Form

$\square$ Keine Dokumentation bzw. Speicherung?

b) Erfolgt eine postoperative Evaluation des neurologischen Status, z.B. im Rahmen der postoperativen anästhesiologischen Visite?

$\square$ Ja, bei jedem Patienten

$\square$ Ja, bei Patienten mit postoperativen neurologischen Komplikationen

$\square$ Nur sporadisch

$\square \mathrm{Nie}$

c) Die Evaluation des neurologischen Status erfolgt

$\square$ individuell durch den Anästhesisten

$\square$ anhand validierter Score-Systeme

$\square$ anhand der AVB's (Anästhesierelevante Verlaufsbeobachtung) auf dem Narkoseprotokoll

d) Bitte geben Sie die Rate neurologischer Komplikationen bei folgenden Operationen an!

ACB $\quad \square<1 \% \quad \square 2-5 \% \quad \square>5 \% \quad \square>10 \% \quad \square>20 \%$

Beating-heart (OPCAB) $\quad \square<1 \% \quad \square 2-5 \% \quad \square>5 \% \quad \square>10 \% \quad \square>20 \%$

Aortenklappenchirurgie $\quad \square<1 \% \quad \square 2-5 \% \quad \square>5 \% \quad \square>10 \% \quad \square>20 \%$

Klappenchirurgie $\quad \square<1 \% \quad \square 2-5 \% \quad \square>5 \% \quad \square>10 \% \quad \square>20 \%$

Doppelklappenersatz $\quad \square<1 \% \quad \square 2-5 \% \quad \square>5 \% \quad \square>10 \% \quad \square>20 \%$

MIC-AKE/R $\quad \square<1 \% \quad \square 2-5 \% \quad \square>5 \% \quad \square>10 \% \quad \square>20 \%$

MIC-MKE/R $\quad \square<1 \% \quad \square 2-5 \% \quad \square>5 \% \quad \square>10 \% \quad \square>20 \%$

$\begin{array}{llllll}\text { MIDCAB } & \square<1 \% & \square 2-5 \% & \square>5 \% & \square>10 \% & \square>20 \%\end{array}$

$\mathrm{ACB}+\mathrm{ACl}-\mathrm{TEA} \quad \square<1 \% \quad \square 2-5 \% \quad \square>5 \% \quad \square>10 \% \quad \square>20 \%$

Kombinationseingriffe $\quad \square<1 \% \quad \square 2-5 \% \quad \square>5 \% \quad \square>10 \% \quad \square>20 \%$

\section{Angaben zur Klinik}

Operationen

$A C B-O p e r a t i o n e n$

OPCAB

Aortenklappenoperationen

Mitralklappenoperationen

Minimal-invasive Techniken

MIC-AKE/R

MIC-MKE/R

MIDCAB

Thorakoabdomineller Aortenersatz

Assist-Sytem Implantationen

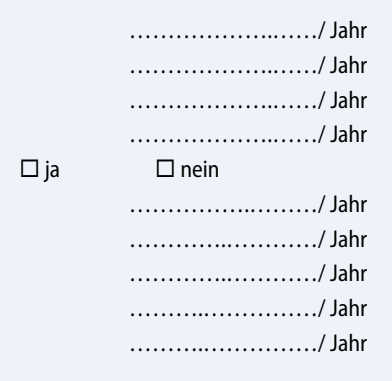

Struktur der Klinik für Anästhesiologie

Oberärzte

Fachärzte

Assistenzärzte

Anzahl.

Anzahl.

Anzahl.

\section{Ausbildungsstand}

$\square$ Die meisten Ärzte sind zertifiziert in einem Neuromonitoring-Verfahren

$\square$ Die Oberärzte sind zertifiziert in einem Neuromonitoring-Verfahren

$\square$ Nur die wenigsten Ärzte sind zertifiziert in einem Neuromonitoring-Verfahren

Fortbildung

$\square$ Es finden regelmässige innerklinische Fortbildungen über Neuromonitoring (-verfahren) statt

$\square$ Es finden regelmässige ausserklinische Fortbildungen über Neuromonitoring (-verfahren) statt

$\square$ Die Fortbildung über Neuromonitoring, (-verfahren) bleibt jedem Arzt selbst überlassen

$\square$ Es finden nur selten Fortbildungen über Neuromonitoring, (-verfahren) statt

$\square$ Es finden keine Fortbildungen über Neuromonitoring, (-verfahren) statt

\section{Abb. $1 \Delta$ Fortsetzung}


- präoperative Evaluation der hirnversorgenden Arterien,

- Perfusionsmanagement während extrakorporaler Zirkulation,

- Erfassung des postoperativen neurologischen Status und

- Aus- und Fortbildung auf dem Gebiet des zerebralen Monitorings.

\section{Design}

Um die relevanten Fragestellungen herauszuarbeiten, wurde eine systematische Literaturrecherche in elektronischen Datenbanken (Medline, Pubmed) durchgeführt. Mit Unterstützung des Arbeitskreises Kardioanästhesie der Deutschen Gesellschaft für Anästhesiologie und Intensivmedizin e.V. (DGAI) wurde daraufhin, im Oktober 2007, ein 26 Punkte umfassender, anonymisierter Fragebogen erstellt.

Die Fragen sollten durch Ankreuzen, auch mehrerer, vorformulierter Aussagen, entsprechend einem Multiple-ChoiceTest, durch den Leiter der jeweiligen kardioanästhesiologischen Abteilung beantwortet werden. Durch Verwendung frankierter Briefumschläge für die Rücksendung der Antwortbögen sollten eine spätere Klinikzuordnung ausgeschlossen und die Anonymität der Teilnehmer gewährleistet werden. Um die Rücklaufquote zu erhöhen, wurde nach Ablauf einer Frist von vier Wochen nach Zustellung an jede Klinik ein Erinnerungsschreiben versandt. Nach einer weiteren Frist von zwei Wochen wurden alle kardioanästhesiologischen Abteilungen nochmals telefonisch kontaktiert und um Rücksendung des Fragebogens gebeten, sofern dies nicht schon geschehen war.

In die Analyse wurden alle bis zum 07.01.2008 zurückgesandten Unterlagen einbezogen. Die statistische Auswertung und die graphische Darstellung der Ergebnisse erfolgten mit den Programmen Microsoft Excel $^{\oplus} 2000$ und Access ${ }^{\circledast} 2000$ für Windows (Microsoft, Redmont, USA). Die Häufigkeit der Antworten wurde mit $\operatorname{dem} \chi^{2}$-Test ausgewertet. Als Signifikanzniveau wurde $\mathrm{p}<0,05$ festgelegt.

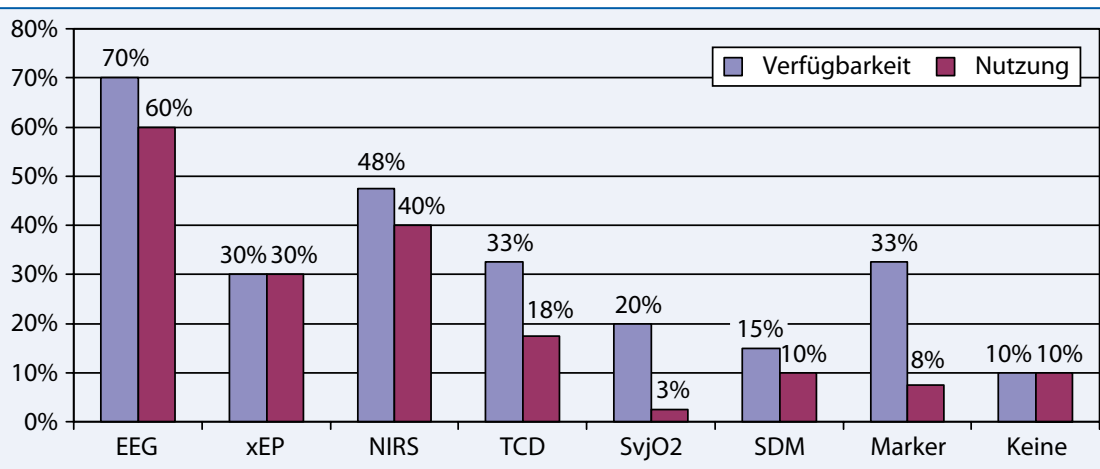

Abb. $2 \Delta$ Verfügbarkeit und Nutzung von Neuromonitoringverfahren. EEG Elektroenzephalographie, $x E P, N I R S$ Nahinfrarotspektroskopie, $T C D$ transkranielle Dopplersonographie, $S_{\mathrm{vj}} \mathrm{O}_{2}$ jugularvenöse Sauerstoffsättigung, SDM Stumpfdruckmessung der A. carotis interna

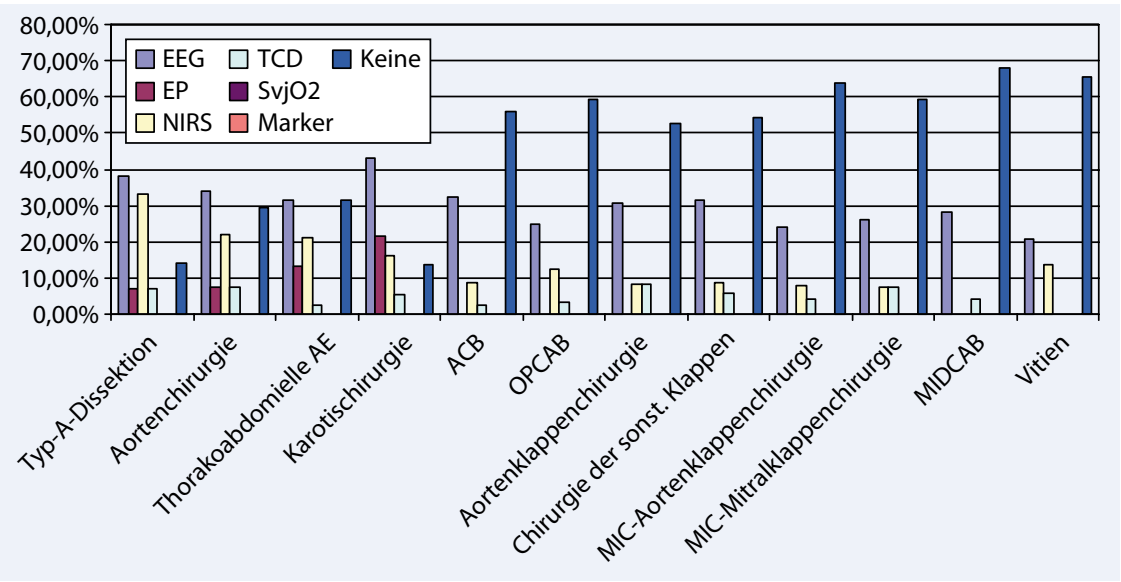

Abb. $3 \Delta$ Anwendung von Neuromonitoringverfahren. $A E$ Aortenersatz, $A C B$ aortokoronarer Bypass, $M I C$ minimalinvasive Chirurgie, MIDCAB "minimally invasive direct coronary artery bypass",

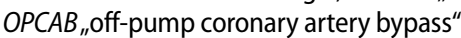

\section{Ergebnisse}

Von den 80 verschickten Fragebögen (• Abb. 1) kamen 44 zurück; dies entspricht einer Rücklaufquote von $55 \%$.

Bei der Frage nach der Verfügbarkeit von Neuromonitoringverfahren in der Abteilung führte das EEG mit 70\%, gefolgt von NIRS (47,5\%), TCD (33\%) und der Ableitung von EP (30\%). Wenn vorhanden, wurden Neuromonitoringverfahren routinemäßig eingesetzt; hierbei war die EEG-basierte Überwachung führend (6o\%). Die NIRS wurde fast immer eingesetzt, wenn vorhanden, während TCD trotz Verfügbarkeit in einem Drittel der Abteilungen nur zu 18\% tatsächlich genutzt wurde (- Abb. 2). Die entsprechenden Verfahren wurden in $45,7 \%$ der Kliniken immer eingesetzt, wenn vonseiten des Patienten oder der Operation eine Indikation bestand. Anschluss der Geräte und Interpretation der Befunde gehörten in $75 \%$ bzw. 90,9\% der Kliniken zum Aufgabengebiet des Anästhesisten. In 98,6\% wurden die gemessenen Parameter intraoperativ ausgewertet.

Bei Typ-A-Dissektionen der Aorta wurden in erster Linie EEG $(38,1 \%)$ und NIRS (33,3\%) als Monitoringverfahren eingesetzt. Von den antwortenden Kliniken verzichteten 14,3\% bei diesen Eingriffen auf jegliche Überwachung der zerebralen Funktion. Bei elektiven Operationen an der thorakalen bzw. thorakoabdominellen Aorta wurden ebenfalls EEG $(34,1 \%$ resp. $31,6 \%)$ und NIRS (21,9\% resp. 21\%) als Präferenz angegeben. Bei diesen Operationen nahmen 30\% der Kliniken keine ZNS-Überwachung vor. In der Karotischirurgie ist das EEG (43,2\%) die führende Methode des Neuromonitorings, gefolgt von EP (21,6\%), NIRS (16,2\%) und TCD (5,4\%). In 13,6\% wurde kein apparatives Hilfsmittel eingesetzt. Ebenso wurde in den meisten Kliniken auf ein ZNS- 


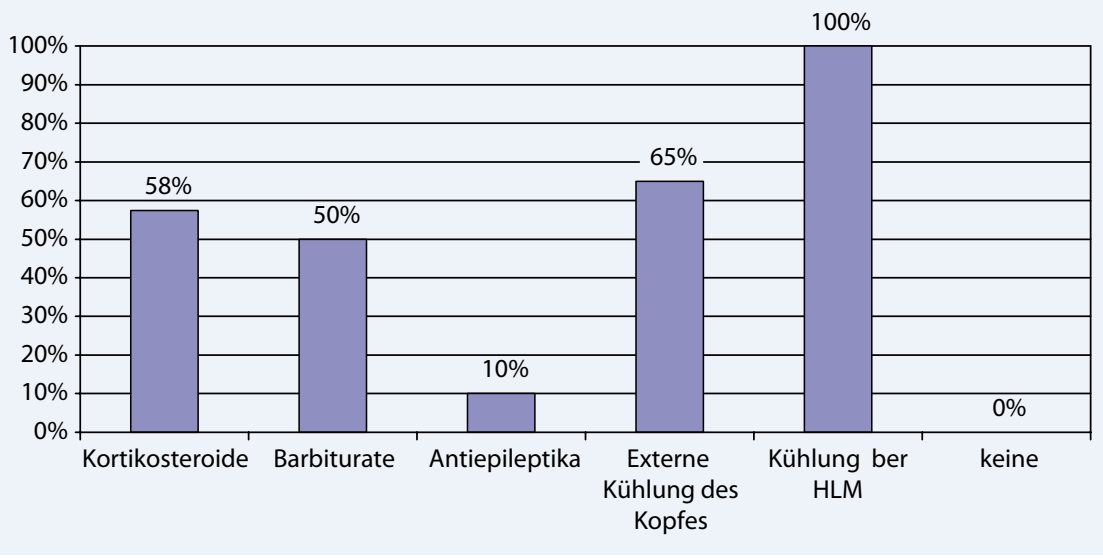

Abb. $4 \Delta$ Frage nach sinnvollen zerebroprotektiven Maßnahmen bei Operationen mit Kreislaufstillstand. HLM Herz-Lungen-Maschine

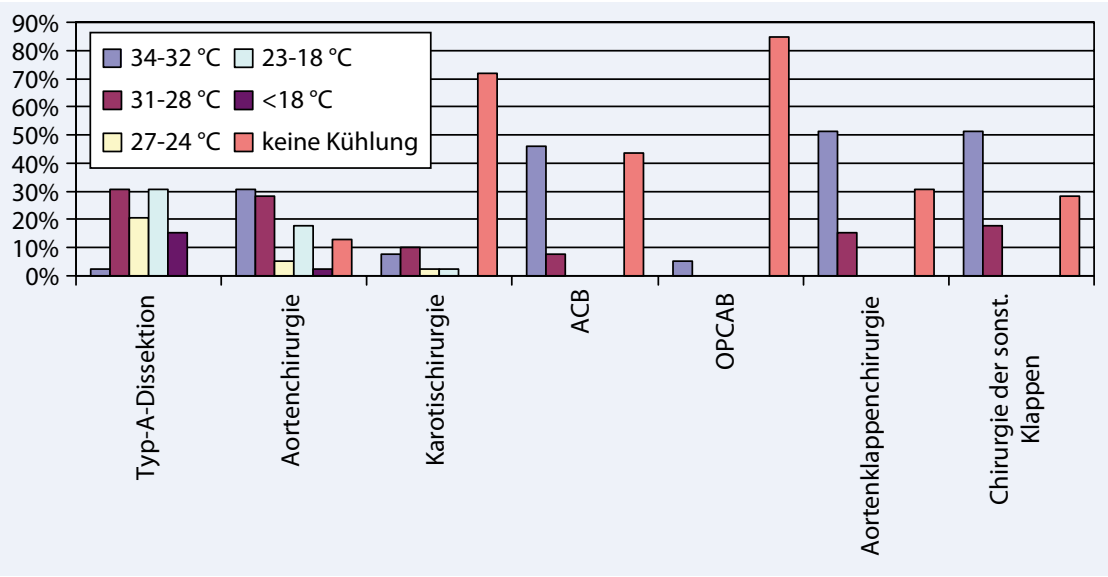

Abb. $5 \Delta$ Frage nach der maximalen Erniedrigung der Körperkerntemperatur.

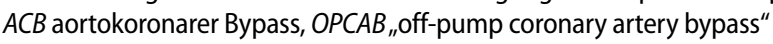

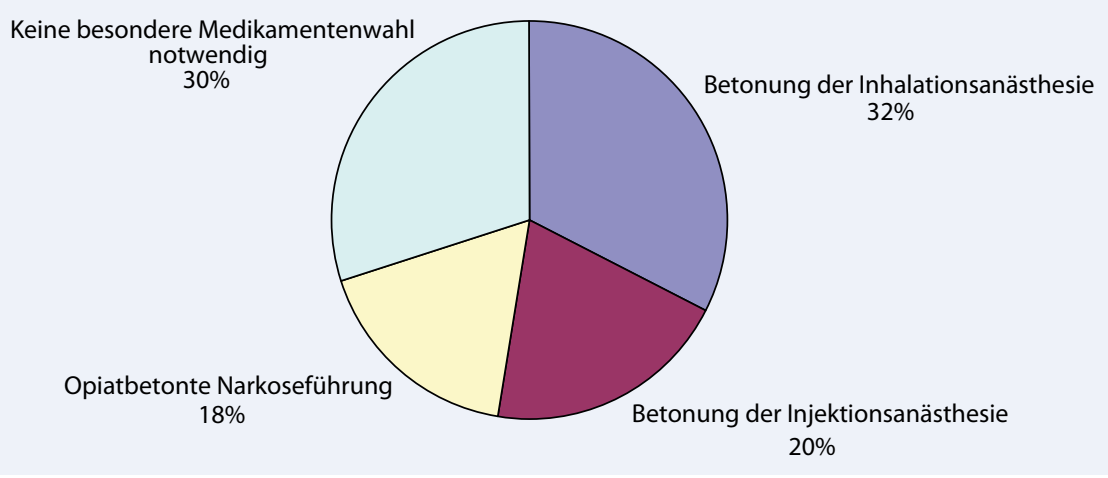

Abb. $6 \triangle$ Frage nach der Existenz einer besonderen Wahl von Narkosemedikamenten für eine neuroprotektive Anästhesie

Monitoring in der Bypass-, Klappen- und minimalinvasiven Chirurgie verzichtet (• Abb. 3).

Als neuroprotektive Maßnahme betrachteten alle Kliniken die Kühlung des Patienten an der Herz-Lungen-Maschine (HLM; 100\%). Zusätzlich wurden die externe Kühlung des Kopfes (65\%), die Gabe von Kortikosteroiden (58\%), Barbituraten (50\%) und Antiepileptika (10\%) als schützende Maßnahmen gegen neuronalen Schaden definiert (• Abb.4). Standardmäßig kühlten $72,5 \%$ der befragten Krankenhäuser ihre Patienten bei Operationen mit Herz-Kreislauf-Stillstand, $37,5 \%$ bei allen Operationen mit HLM. In 37,5\% der
Abteilungen wurde individuell über eine iatrogene Hypothermie entschieden. Während bei Bypass- und Herzklappenoperationen über eine mäßige Hypothermie (bis maximal $32^{\circ} \mathrm{C}$ ) Einigkeit herrschte, waren die Meinungen über die notwendige Temperatur bei Typ-A-Dissektionen der Aorta und in der Aortenchirurgie geteilt (• Abb. 5). Bei der Frage nach einer besonderen Kombination von Narkotika zur Neuroprotektion kreuzten $32,5 \%$ der teilnehmenden Kliniken die Inhalationsanästhesie (Favorit: Sevofluran $76,5 \%), 20 \%$ die total intravenöse Anästhesie (Favoriten: Propofol und Barbiturate mit jeweils $46,2 \%$ ) an. $30 \%$ der Befragten hielten keine besondere Medikamentenauswahl für eine neuroprotektive Anästhesie für notwendig ( $\bullet$ Abb. 6).

Unter normothermen Bedingungen entsprach in $84,6 \%$ der Kliniken der HLM-Fluss dem errechneten Herzzeitvolumen (HZV) des Patienten. Über den gleichzeitig anzustrebenden mittleren arteriellen Blutdruck (MAP) waren die Befragten uneinig. Während $43,9 \%$ einen MAP von $60-70 \mathrm{mmHg}$ für adäquat hielten, favorisierten $41,5 \%$ einen MAP zwischen 50 und $60 \mathrm{mmHg}$ und $2,4 \%$ einen MAP $>70$ mmHg. Es strebten $12,2 \%$ der Kliniken keinen bestimmten Perfusionsdruck unter Normothermie an. Bei Körpertemperaturen zwischen 28 und $34^{\circ} \mathrm{C}$ sollte nach $82 \%$ der teilnehmenden Häuser der HLM-Fluss nicht reduziert werden und dem errechneten HZV entsprechen. Unter $28^{\circ} \mathrm{C}$ hielten $46 \%$ der Studienteilnehmer einen HLM-Fluss wie unter Normothermie für adäquat, während $46 \%$ den HLM-Fluss unter das errechnete HZV reduzierten bzw. 8\% einen HLMFluss über dem errechneten HZV einstellten. Bei unter $18^{\circ} \mathrm{C}$ wurde der HLM-Fluss unter das errechnete HZV gesenkt (70\%), während in $27 \%$ der Kliniken normotherme und in $3 \%$ höhere Flussraten gefahren wurden. Der bevorzugte MAP unter Hypothermie lag zwischen 50 und $60 \mathrm{~mm}$ $\mathrm{Hg}(59 \%)$.

Eine präoperative Untersuchung hirnversorgender Arterien erfolgte in $82 \%$ der teilnehmenden Kliniken routinemäßig, in 12\% nur bei neurologischer Symptomatik. Jeweils $2 \%$ der Kliniken führten eine präoperative Untersuchung nur vor entsprechender Operation (z. B. Ka- 
rotisendarteriektomie), selten bzw. nie durch (• Abb. 7). Die am häufigsten verwendete Methode war die Duplexsonographie (90\%). Andere Verfahren, wie z. B. die TCD spielten in der präoperativen Routineuntersuchung eine untergeordnete Rolle (• Abb. 8). Die Anordnung der apparativen Untersuchung oblag in erster Linie dem Operateur $(62,5 \%)$. Die Wahl des geeigneten intraoperativen Neuromonitorings traf der Anästhesist in $84 \%$, der Chirurg in $13 \%$ und der Stationsarzt in 3\% der Kliniken.

Die Dokumentation der Ergebnisse des zerebralen Neuromonitorings erfolgte in den meisten Fällen im Narkoseprotokoll (47\%), seltener in elektronischer Form $(21,5 \%)$, separat in eigenem Vordruck (19,6\%) oder gar nicht (11,9\%). Eine postoperative Erhebung des neurologischen Status wurde meistens im Rahmen der postoperativen Visite (42,5\%) und in $77,5 \%$ der Fälle durch individuelle Einschätzung des Anästhesisten durchgeführt. Validierte Score-Systeme wurden in $17,5 \%$ der Kliniken angewandt. Bei $32,5 \%$ der befragten Abteilungen wurde der postoperative neurologische Status nur bei Patienten mit neurologischen Komplikationen erhoben. Es gaben 7,5\% an, nie eine diesbezügliche klinische Untersuchung durchzuführen.

Fortbildungen zum Thema Neuromonitoring organisieren regelmäßig 32,5\% der Kliniken, nur selten $40 \%$. In 37,5\% der befragten Häuser bleibt die Verantwortung für die Weiterbildung auf diesem Gebiet jedem Arzt individuell überlassen.

\section{Diskussion}

\section{Konzept des multimodalen Neuromonitorings}

Eine Vielzahl von wissenschaftlichen Arbeiten betont seit mehr als zehn Jahren die Notwendigkeit der Überwachung des ZNS während kardiochirurgischer Eingriffe. Es ist mittlerweile klar, dass das Auftreten von PNCD eng an das Patientengut [40] und, u. a. als Folge von Embolisation, Hypo- und Hyperperfusion bzw. inadäquatem Temperaturmanagement, an prozedurbedingte spezifische Risiken herzchirurgischer Interventionen gebunden ist [53]. Die Anwendung von Neu-

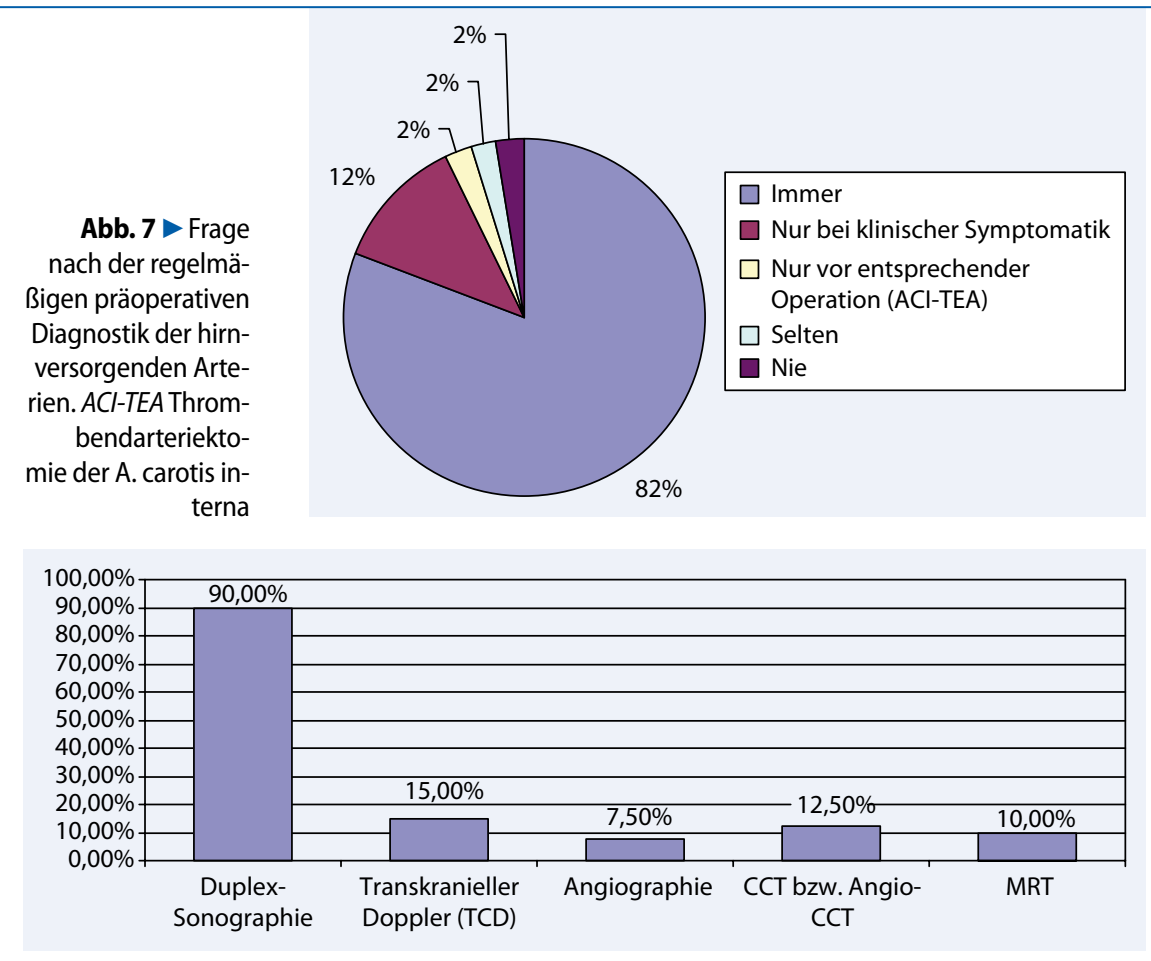

Abb. 8 A Untersuchungsmethoden, TCD transkranielle Dopplersonographie, CCT kraniale Computertomographie, MRT Magnetresonanztomographie

romonitoring vermag solche Komplikationen zu senken [41] und Kosten zu reduzieren [32]. Weil jedoch kein Verfahren allein eine optimale und ganzheitliche intraoperative Überwachung des Patienten erlaubt, wird entsprechend dem Konzept des „multimodalen Neuromonitorings" empfohlen, zerebrale Funktionen mithilfe mehrerer unterschiedlicher Verfahren (EEG, EP, NIRS, TCD; [24, 30]) zu überwachen. Wie weit allerdings dieses Konzept Eingang in die tägliche Praxis kardioanästhesiologischer Abteilungen in Deutschland gefunden hat, ist bisher nicht bekannt. Unklar ist weiterhin, welche zerebrale Überwachungsmaßnahme bei welchem Eingriff benutzt und welche Art von Neuroprotektion angewendet wird.

\section{Rücklaufquote der Erhebung}

Nur knapp die Hälfte der angefragten Kliniken hat den Fragebogen beantwortet und zurückgeschickt. Über die Gründe der eher bescheidenen Rücklaufquote kann nur spekuliert werden. Infrage kommen u. a. mangelnde Zeit der Befragten, ein zu komplex gestalteter Fragebogen, geringer Stellenwert des angesprochenen Themenkomplexes in der jeweiligen kardioanästhesiologischen Abteilung u.v.m.
Ein nationaler bzw. internationaler Vergleich der Umfrageergebnisse ist angesichts fehlender Parallelen nicht möglich. Recherchen in elektronischen Datenbanken lieferten eine einzige Studie, bei der Neuromonitoring und Neuroprotektion anhand eines Fragebogens, wenn auch nur als Sekundärparameter, untersucht wurden: Bei einer Rücklaufquote von $50,7 \%$ stellten die Autoren fest, dass 90\% der befragten Anästhesisten während Karotisendarteriektomien ein intraoperatives Neuromonitoring verwenden; hierbei wurde zu 6o\% die EEG-basierte Überwachung bevorzugt [5].

\section{Elektroenzephalographie}

Die Bevorzugung des EEG als Neuromonitoring konnte im Rahmen dieser Untersuchung bestätigt werden. Verfügbarkeit und Einsatzhäufigkeit des Verfahrens in deutschen kardioanästhesiologischen Abteilungen übertrafen bei Weitem andere Methoden des apparativen Neuromonitorings. Dabei war die Nutzung des EEG nicht nur auf Operationen der A. carotis beschränkt. Das Verfahren erfreute sich vielmehr einer breiten Anwenderbasis bei allen hier erfragten kardiochirurgischen Prozeduren. 
Das EEG ist ein nichtinvasives, kontinuerliches Verfahren mit schneller Änderung von Amplitude und Frequenz bei Abfall des zerebralen Blutflusses unter $22 \mathrm{ml} / 100$ g Hirngewebe pro min [24]. Eine enge Beziehung zwischen simultanen Veränderungen und regionalem zerebralen Blutfluss sowie neurologischen Ereignissen konnte jedoch nur für die Karotisendarteriektomie gezeigt werden [56]. Auch spezifische Nachteile der Technik sind hinreichend bekannt:

- Eine aussagekräftige Datenanalyse des Roh-EEG ist komplex und erfordert erfahrenes Personal.

- Ischämien in subkortikalen Hirnregionen und im Rückenmark werden nicht erfasst.

- Die registrierten EEG-Veränderungen sind häufig unspezifisch, sodass zerebrokortikale Depressionen, verursacht durch Hypoperfusion oder Sauerstoffimbalance, nicht notwendigerweise von Veränderungen unterschieden werden können, die durch Anästhetika oder Hypothermie induziert worden sind.

- Eine Verschlechterung der Signalqualität durch elektrische Interferenzen (Rollerpumpe der HLM, Elektrokauter, „pacemaker“, Wärmegeräte) oder „shivering“ kann trotz modernster Gerätetechnik nicht immer verhindert werden.

Was sind also die Gründe, die trotz der Nachteile, die häufige Anwendung des EEG bei kardiochirurgischen Operationen in Deutschland erklären bzw. rechtfertigen?

Das EEG hat ohne Zweifel spezifische Vorteile gegenüber anderen Messtechniken der Hirnfunktion: Die Onlineüberwachung der elektrischen Hirnaktivität prädestiniert das Verfahren für die Chirurgie der Aorta und der supraaortalen Gefäße. Bei der Vielfalt von Klemmund Perfusionstechniken können Phasen der zerebralen Minderdurchblutung („low flow") schnell erkannt und therapiert werden. Das Auftreten einer „burst suppression" (Sistieren der hirnelektrischen Aktivität) im EEG als Zeichen einer homogenen und adäquaten Hirnkühlung vor einem operationstechnisch erforderlichen
Kreislaufstillstand kann mit keinem anderen Verfahren erfasst werden [55].

Die führende Rolle des EEG als Neuromonitoring in dieser Untersuchung scheint jedoch zusätzlich durch das Studiendesign per se entstanden zu sein. Bei der Frage nach Neuromonitoringverfahren ist nicht zwischen den verschiedenen EEG-basierten Verfahren unterschieden worden. Es ist wahrscheinlich, dass neben der Ableitung des Roh-EEG als eigentliche zerebrale Überwachungsmethode auch quantitative EEG-Formen (QEEG), z. B. bispektraler Index (BIS), als Neuromonitoringverfahren verstanden und angegeben wurden. Eventuell ist dadurch eine Art systematischer Fehler entstanden, sodass die hohe Repräsentanz des EEG als Neuromonitoringverfahren u. U. mit der Verbreitung des BIS als Monitoringtechnik der Narkosetiefe in der heutigen Ära der „Fast-track-Anästhesie” [11, 58] zu erklären ist.

Zwar reflektieren ischämiebedingte Änderungen des QEEG Änderungen im konventionellen EEG [4]; eine Gleichsetzung beider Verfahren ist jedoch nicht gerechtfertigt. Die Detektion kortikaler Ischämien $[1,13]$, zerebraler Hypoperfusion [39] und Gasembolisation $[16,57] \mathrm{mit}$ QEEG ist allein in nichtrandomisierten Studien mit kleiner Teilnehmerzahl bzw. in Fallberichten gelungen. Wie weit diese Untersuchungen jedoch als repräsentativ gelten können und ob EEG-basiertes Monitoring in der Herzchirurgie dem heutigen Standard entspricht, ist angesichts zahlreicher Studien mit negativer $([23,27])$ sowie positiver $([26,30])$ Korrelation zwischen EEG und zerebraler Ischämie immer wieder Gegenstand neuer Diskussionen. Eine aktuelle Studie bei wachen Patienten während Operationen an der A. carotis interna zeigt, dass zerebrale Ischämien in nur 59,4\% der Fälle festgestellt werden können [27].

Angesichts der kontroversen Studienlage und der Tatsache, dass eine korrekte EEG-Registrierung durch zahlreiche, gerade im herzchirurgischen OP anzutreffende Interferenzen beeinträchtigt werden kann, ist fraglich, ob die Verwendung des EEG bzw. des QEEG als alleiniges bzw. bevorzugtes Überwachungsgerät zur zerebralen Ischämiediagnostik in der Kardioanästhesie gerechtfertigt ist.

\section{Nahinfrarotspektroskopie}

Wie die Umfrage zeigte, sind andere Verfahren der zerebralen Überwachung in Deutschland durchaus verbreitet, auch wenn sie in weniger als 50\% der Kliniken verfügbar sind. Die festgestellte hohe Korrelation zwischen Verfügbarkeit und tatsächlicher Nutzung von NIRS (48\% bzw. $40 \%$ ) und EP (30\% bzw. 30\%) hängt am wahrscheinlichsten mit der bewussten Anschaffung dieser Apparaturen für spezielle klinische Fragestellungen zusammen.

Die NIRS verdient in der zerebralen Ischämiediagnostik einen festen Stellenwert vorzugsweise als bilaterales Monitoringverfahren seitengetrennter Perfusion [35]. Dies wird durch mehrere Studien und auch durch die Ergebnisse dieses Fragebogens bestätigt: Das Verfahren wurde vorzugsweise in der Aorten- und der Karotischirurgie eingesetzt, während sein Einsatz bei aortokoronaren Bypass(ACB-) bzw. Herzklappeneingriffen gering war. Grundlage der Messung bildet die gute Korrelation zwischen dem durch NIRS ermittelten Abfall der regionalen Sauerstoffsättigung $\left(\mathrm{rSO}_{2}\right)$ und dem postoperativen Auftreten von PNCD [6o]. Die $\mathrm{rSO}_{2}$-Bestimmung kann und soll dabei nicht konventionelle Parameter der adäquaten (Hirn-)Perfusion wie arterielle, venöse, gemischt-venöse Sauerstoffsättigung, Parameter des Säure-Basen-Haushalts oder das HZV ersetzen. Sie gilt vielmehr als ein den intraoperativen, individuellen Trend der zerebralen Sauerstoffversorgung anzeigendes Verfahren, wobei auch Kritikpunkte [extrakranielle Kontamination der Werte, fehlende Detektion posteriorer Hirnminderdurchblutung, Beeinflussung durch Hämodilution und endexspiratorische Kohlensäure- $\left(\mathrm{CO}_{2}\right)$ Konzentration] berücksichtigt werden müssen [12]. Im Rahmen des multimodalen Neuromonitorings soll die NIRS zu einer rechtzeitigen Korrektur entgleister (zerebraler) Perfusionsparameter [MAD, HLM-Fluss, arterieller Kohlensäurepartialdruck $\left(\mathrm{p}_{\mathrm{a}} \mathrm{CO}_{2}\right)$, Hämatokrit] beitragen.

\section{Ableitung evozierter Potenziale}

Evozierte Potenziale wurden, laut Umfrage, als das dritthäufigste Neuromoni- 
toringverfahren angegeben; hierbei beschränkte sich ihre Anwendung auf Eingriffe an der thorakalen bzw. thorakoabdominellen Aorta und der A. carotis. Diese Indikationen entsprechen dem aktuellen wissenschaftlichen Kenntnisstand. Die Ableitung akustisch evozierter Potenziale ist $u$. a für das Monitoring einer adäquaten zerebralen Hypothermie bzw. der Vermeidung von Hyperthermie (Eingriffe mit Herz-Kreislauf-Stillstand) sinnvoll, während somatosensorisch und motorisch evozierte Potenziale in erster Linie als Verfahren zur Aufdeckung zerebraler sowie spinaler Ischämien (z. B. Karotischirurgie, thorakoabdomineller Aortenersatz) propagiert werden [52].

\section{Transkranielle Dopplersonographie}

Laut Umfrage ist die TCD in Deutschland sowohl als intraoperatives Neuromonitoring als auch als diagnostisches Hilfsmittel vor einer Herzoperation verfügbar. Während sie bei der präoperativen Untersuchung der Hirngefäße den zweiten Platz, hinter der Duplexuntersuchung, belegt, scheint ihre intraoperative Anwendung nicht sehr verbreitet $\mathrm{zu}$ sein: Bei einer Verfügbarkeit von knapp einem Drittel der Abteilungen wird sie ca. nur von der Hälfte der entsprechend ausgerüsteten Kliniken in der täglichen Routine benutzt. Dabei ist das Einsatz- gebiet der TCD vielfältig und gerade in der modernen Kardiochirurgie mit steigender Anzahl älterer Patienten und minimalinvasiver Eingriffe von besonderem Interesse. Als einziges nichtinvasives und kontiniuerliches Neuromonitoringverfahren ist sie in der Lage, intraoperative Änderungen der Hirnperfusion als „real time cerebral blood flow“ zu messen $[45,58]$. So ist die TCD insbesondere in Situationen angebracht, in denen eine adäquate Hirnperfusion nicht mit hinreichender Sicherheit erfasst werden kann (z. B. Typ-A-Dissektion, Aortenchirurgie, selektive/retrograde Hirnperfusionstechniken, „Off-pump“-Bypasschirurgie, „low flow" der HLM). Die Technik ist ebenfalls geeignet, die unmittelbaren Konsequenzen anästhesiologischer Interventionen (Kreislaufmanagement, Volumen- und Vasopressorengabe) sowie chirurgischer Maßnahmen (Aortenkanülierung, Gefäßligatur) zu überwachen und in intraoperative Entscheidungen ohne zeitlichen Verlust einzubeziehen. Als Besonderheit gelten die quantitative und die qualitative Erfassung embolischer Ereignisse [25]. Die Detektion von "high-intensity transient signals“ kann vor allem bei Eingriffen mit hohem Embolierisiko (Herzklappen-, Bypass-, minimalinvasive Chirurgie, Aortenatherom, , assist device“) zur Optimierung von Perfusions- [14], Kanülie- rungsmaßnahmen [36] bzw. des Gerinnungsmanagements [44] beitragen.

Angesichts der erwähnten Studienlage ist der seltene Einsatz des TCD-Monitorings in Deutschland nicht verständlich, aber wahrscheinlich u. a durch die TCDTechnik per se zu erklären. Während NIRS und EP durch Positionierung der Elektroden bereits nach wenigen Minuten verwertbare Ergebnisse liefern, ist die Nutzung der TCD aus folgenden Gründen komplexer:

- Die Technik erfordert einen erhöhten Ausbildungsaufwand.

- Die Auffindung des temporalen Knochenfensters, das für die intraoperative zerebrale Gefäßanlotung unerlässlich ist, misslingt bei 10-20\% der (älteren) Patienten.

- Die Erhebung valider Daten ist nur durch gut fixierte Ultraschallsonden am Kopf möglich. Dies kann je nach Untersucher und Sondenfixierungsbesteck erhebliche (OP-)Zeit (und Kosten!) sowie Geduld in Anspruch nehmen.

\section{Hypothermie}

Die zerebroprotektive Wirkung der tiefen Hypothermie $\left(28-18^{\circ} \mathrm{C}\right)$ bei Operationen mit Herz-Kreislauf-Stillstand ist unbestritten [19]. Deshalb ist es auch nicht überraschend, wenn diese Maßnahme von al-

\title{
Hier steht eine Anzeige.
}

\author{
望 Springer
}


len befragten Klinken angegeben wurde. Dass über die Einstellung einer milden $\left(34-32^{\circ} \mathrm{C}\right)$ bis mäßigen $\left(32-28^{\circ} \mathrm{C}\right) \mathrm{Hy}-$ pothermie Uneinigkeit herrscht, zeigt jedoch die Frage nach dem Temperaturmanagement in Abhängigkeit vom jeweiligen Eingriff. Während die erfragte Angabe verschiedener Zieltemperaturen bei Typ-A-Dissektion und in der Aortenchirurgie wegen der Vielzahl pathologischer Gefäßbefunde bzw. operativer Techniken verständlich ist, spiegelt die Temperatureinstellung in der Bypass- und Herzklappenchirurgie die unterschiedlichen Ansichten wider.

Fest steht: Die tiefe Hypothermie ist bei Eingriffen mit Herz-Kreislauf-Stillstand wegen der erhöhten Toleranz des neuronalen Gewebes gegenüber ischämischer Schädigung unbedingt indiziert. Die milde Hypothermie erscheint ebenfalls günstig: Die Reduktion des Hirnstoffwechsels $\left(6-7 \% /{ }^{\circ} \mathrm{C}\right.$-Temperaturabfall) und weitere neuroprotektive Effekte [Blockierung der Glutamatausschüttung, Reduktion des Kalziumeinstroms, Verminderung der Proteinkinase-C-Aktivität, beschleunigte Proteinbiosynthese, Unterdrückung der Bildung freier Radikale und der Stickstoffmonoxid- (NO-)Synthetaseaktivität] rechtfertigen ihre Anwendung im Bereich der Herzchirurgie [22]. Wie die Ergebnisse zeigen, wird die milde Hypothermie in Deutschland ebenfalls praktiziert: Bei über $40 \%$ der ACB- und über $50 \%$ der Herzklappenoperationen wurden die $\mathrm{Pa}$ tienten geringgradig gekühlt.

Während der positive Effekt einer milden Hypothermie gegen eine ischämische Schädigung des Gehirns gesichert ist [47], ist die protektive Wirkung der Hypothermie auf die neurokognitive Funktion nach herzchirurgischem Eingriff nicht belegt [21]. Der Grund für die teilweise widersprüchlichen Ergebnisse liegt nach aktueller Studienlage an einer überschießenden postinterventionellen Hyperthermie der Patienten auf über $37^{\circ} \mathrm{C}$. Neben der stärkeren axonalen Schädigung der Neuronen [38] konnte eine erhöhte Inzidenz neurokognitiver Dysfunktionen bis zu sechs Wochen nach herzchirurgischem Eingriff bei schnellem Wiedererwärmen nachgewiesen werden [20]. Angaben zu postinterventioneller Temperatur und zum Auftreten von Hyperthermie wurden im Rahmen dieser Studie nicht erfragt. Wiederaufwärmstrategien nach kardiochirurgischem Eingriff können deshalb nicht erörtert werden. Nach Angaben der Literatur steht jedoch fest: Hypothermie ist nur dann von Vorteil, wenn sie postinterventionell nicht zur überschießenden Wiederaufwärmung führt!

Der Ort der Temperaturmessung ist für das Temperaturmanagement mindestens genauso wichtig wie die Zieltemperatur. Blasen- und ösophageale Temperatur können, je nach Aufwärm- bzw. Kühlphase, um $4^{\circ} \mathrm{C}$ resp. $2^{\circ} \mathrm{C}$ niedriger bzw. höher als die jugularvenöse Temperatur liegen [48], die der beste Surrogatparameter für die zerebrale Temperatur darstellt [54]. Leider war der Ort der Temperaturmessung nicht Gegenstand des Fragebogens, sodass Aussagen zu Häufigkeitsverteilung und Präferenz der Temperaturmessorte nicht getroffen werden können.

\section{Pharmakologische Neuroprotektion}

Wie diese Umfrage zeigt, ist die Praxis der pharmakologischen Neuroprotektion mit Barbituraten, Antiepileptika und Kortikosteroiden in Deutschland durchaus verbreitet. Eine standardisierte Applikation der Substanzen bei bestimmten intraoperativen Konstellationen konnte im Rahmen der Fragebogenaktion jedoch nicht ermittelt werden. Dies entspricht den Ergebnissen einer Studie von Dewhorst et al. [10], die mangels nationaler und internationaler Vorgaben keinen praktikablen Einsatz oben erwähnter Pharmaka in Großbritannien zur Zerebroprotektion während eines tief hypothermen Kreislaufstillstands erkennen konnte.

Daten, die die Anwendung dieser Medikamente im Sinne eines prophylaktischen neuroprotektiven Effektes in der Kardiochirurgie eindeutig stützen würden, gibt es nicht [33]. Eine Studie von Nussmeier et al. [46], bei der ein verbessertes neurologisches Outcome nach Barbituratapplikation festgestellt wurde, konnte Jahre später nicht verifiziert werden [59]. Zurzeit gibt es keine klinische Studie, die eine verringerte Mortalitätsrate oder bessere neurologische Ergebnisse aufgrund einer Barbituratapplikation zur Hirnprotektion beweist. Für Kortikoste- roide konnte sogar, u. a. wegen der induzierten Hyperglykämie, ein verschlechtertes neurologisches Outcome nachgewiesen werden, sodass deren Zufuhr für die Hirnprotektion in der Kardiochirurgie nicht empfohlen werden kann [22]. Dass in dieser Umfrage Barbiturate und Kortikosteroide den höchsten Wert aller pharmakologischen Interventionen erreicht haben, ist zwar erstaunlich, stimmt jedoch mit den Ergebnissen anderer Untersucher überein: In einer Studie, in der die Praxis der Neuroprotektion in der Neuroanästhesie untersucht wurde, erreichten Barbiturate den ersten und Kortikosteroide den dritten Platz unter den infrage kommenden Substanzen [29]. Bei der Suche nach Gründen für die Anwendung dieser Substanzen in deutschen kardiochirurgischen Abteilungen kann nur spekuliert werden. Es ist anzunehmen, dass zumindest die Anwendung der Kortikosteroide auf deren antiinflammatorischen Eigenschaften und damit auf der Reduktion ischämievermittelter Hirnschäden beruht $[6,7]$. Dieser Beweis konnte jedoch, bis auf die Behandlung der isolierten Rückenmarkverletzung [3], klinisch bisher nicht erbracht werden.

Propofol und volatile Anästhetika sind in tierexperimentellen Studien neuroprotektiv, können jedoch nur milde oder mäßige ischämische neuronale Schädigungen reduzieren [28]. Der Wirkmechanismus scheint dabei weniger die Reduktion der zerebralen Metabolisierungsrate von Sauerstoff zu sein, sondern die positive Modulation pathophysiologischer Kaskaden, die erst durch die Ischämie in Gang gesetzt werden $[2,17,18,34]$. In diesem Kontext ist die konsequente Nutzung ausgewählter Anästhetika durchaus sinnvoll, wobei deren Anwendung im Rahmen einer multimodalen neuroprotektiven Strategie nur als supportive Maßnahme empfohlen werden kann $[19,34]$.

\section{Fluss der Herz-Lungen-Maschine und mittlerer arterieller Druck}

Essenziell für ein optimales postoperatives neurologisches Ergebnis ist die Einstellung von HLM-Flow und MAP während der extrakorporalen Zirkulation. Ähnlich wie die Wahl der optimalen Zieltemperatur ist die Steuerung dieser 
Parameter von einer Vielzahl von Variablen abhängig.

Um trotzdem einen Trend feststellen zu können, wurde die Fragestellung nach der adäquaten Einstellung von Flow und MAP nur auf die aktuelle Patiententemperatur als einzige Variable bezogen. Wie das Ergebnis der Umfrage jedoch zeigt, ist die Abstimmung obiger Parameter nicht auf die Variable „Temperatur” zu reduzieren. Neben individueller Überzeugung des Operationsteams bzw. des Kardiotechnikers können Parameter wie z. B. Patientenalter, Komorbiditäten, Hämodilution, Low flow nicht unberücksichtigt gelassen werden. Somit überrascht die festgestellte große Diskrepanz zwischen deutschen kardioanästhesiologischen Abteilungen nicht. Es ist fraglich, ob die Angabe eines „Referenz-Flows” bzw. -MAP überhaupt sinnvoll ist. Untersuchungen auf diesem Gebiet kommen immer mehr zu dem Ergebnis, dass sich die Einstellung einer adäquaten (Hirn-)Perfusion an sensitiven, individuellen Indikatoren orientieren sollte. Hierzu gehören die Messung der (zerebralen) Blutlaktatkonzentration (vorzugsweise über Bulbuskatheter) bzw. die Messung des $\Delta \mathrm{p}_{\mathrm{av}} \mathrm{CO}_{2}[8,9,49,50,51]$ und nicht zuletzt der Einsatz zerebraler Monitoringverfahren [43].

\section{Erhebung des neurologischen \\ Status sowie Fort- und Weiterbildung der Anästhesisten}

Als Bestandteil des ganzheitlichen Patientenmonitorings wurden ebenfalls die präund die postoperative Erhebung des neurologischen Status erfragt. Die erwähnten Neuromonitoringverfahren werden präoperativ in diesem Gebiet nicht eingesetzt. Dies überrascht nicht, da mit der Dopplersonographie eine einfache und nichtinvasive Methode zur Beurteilung von Stenosen der hirnversorgenden Arterien möglich ist. Die postoperative Erhebung erfolgt laut Fragebogen sinnvollerweise im Rahmen der postoperativen Visite; hierbei genügt in überwiegender Zahl der Kliniken die individuelle Einschätzung des neurologischen Status bei der Prämedikationsvisite durch den Anästhesisten. Dies steht im Widerspruch zur Angabe über die Organisation von Fortbildungen auf dem Gebiet des Neuromo- nitorings. Die Bereitschaft und die Initiative zu Fortbildungen zu diesem Thema waren nach Ergebnissen unserer Umfrage gering.

Angesichts des Fehlens entsprechender Vergleiche in nationalen und internationalen Datenbanken kann über diesbezügliche Ergebnisse nur spekuliert werden. Nach Ansicht der Autoren gehört die postoperative Erhebung des neurologischen Status, ebenso wie das intraoperative zerebrale Monitoring, zum erforderlichen Standard der kardioanästhesiologischen Versorgung. Erst durch validierte Fragebögen (z. B. „Wechsler Memory Scale“, Hamburg-Wechsler-Intelligenztest) sowie eine hohe fachliche Kompetenz können das Neuromonitoring optimiert und folglich die negativen sozioökonomischen Auswirkungen von PNCD gesenkt werden.

\section{Fazit für die Praxis}

Die vorliegende Studie zeigt, dass Neuromonitoring und Neuroprotektion in der Kardioanästhesie in Deutschland im Jahr 2008 nicht standardisiert sind. Die EEGbasierte zerebrale Überwachung ist führend und wird bei allen erfragten kardiochirurgischen Eingriffen benutzt. Fraglich bleibt, warum fast die Hälfte deutscher Kliniken bei Operationen an der Aorta bzw. OPCAB- oder Herzklappeneingriffen gar kein Neuromonitoring verwendet und warum neue Methoden des zerebralen Monitorings, wie NIRS oder TCD, bislang keine breite Anwenderbasis gefunden haben.

Im Rahmen des seit 2004 durch Edmonds [15] bzw. Murkin [42] propagierten Konzeptes des „multimodalen Neuromonitorings" ist gerade in der Kardiochirurgie eine zerebrale Überwachung erforderlich, um auf Situationen mit kritischer zerebraler Perfusion zeitgerecht und adäquat reagieren zu können. Eine Ergänzung apparativer Verfahren durch differenzierte präoperative Untersuchung und klinische Beurteilung des Patienten bzw. durch intraoperative Überwachung des Pupillenstatus ist sinnvoll und einfach durchzuführen. Angesichts der steigenden Anzahl multimorbider kardiochirurgischer Patienten, der Zunahme minimalinvasiver Operationstech- niken und der Kostenintensität postoperativer neurologischer sowie neuropsychologischer Defizite im Gesundheitswesen scheinen die vermehrte Nutzung der vorhandenen Möglichkeiten des Neuromonitorings und die intensive Weiterbildung eine Conditio sine qua non zu sein!

\section{Korrespondenzadresse}

\section{Dr. G. Erdös D.E.A.A.}

Inselspital, Universitätsklinik für Anästhesiologie und Schmerztherapie

3010 Bern

Schweiz

Gabor.Erdoes@insel.ch

Danksagung. Die Autoren bedanken sich bei antwortenden Herzzentren, die mit ihrer Mitarbeit diese Arbeit erst möglich gemacht haben.

Interessenkonflikt. Der korrespondierende Autor gibt an, dass kein Interessenkonflikt besteht.

\section{Literatur}

1. Arom K, Cohen D, Strobl F (1989) Effect of intraoperative intervention on neurological outcome based on electroencephalographic monitoring during cardiopulmonary bypass. Ann Thorac Surg 48:476-483

2. Bickler PE, Fahlmann CS (2006) The inhaled anesthetic, isoflurane, enhances $\mathrm{Ca} 2+-$ dependent survival signaling in cortical neurons and modulates MAP kinases, apoptosis proteins and transcription factors during hypoxia. Anesth Analg 103:419-429

3. Bracken MB, Shepard MJ, Collins WF et al (1990) A randomized, controlled trial of methylprednisolone or naloxone in the treatment of acute spinal cord injury. Results of the Second National Acute Spinal Cord Injury Study. N Engl J Med 1322:14051411

4. Chabot RJ, John ER, Prichep LS et al (1993) Real-time multichannel quantitative EEG monitoring. In: Willner A (ed) Cerebral damage before and after cardiac surgery. Kluwer, Oordecht, pp 163-181

5. Cheng MA, Theard MA, Tempelhoff R (1997) Anesthesia for carotid endarterectomy: a survey. J Neurosurg Anesthesiol 3:211-216

6. Chopp M, Zhang RL, Chen H et al (1994) Postischemic administration of an anti-Mac-1 antibody reduces ischemic cell damage after transient middle cerebral artery occlusion in rats. Stroke 25:869875, discussion 75-76

7. Clark RK, Lee EV, White RF et al (1994) Reperfusion following focal stroke hastens inflammation and resolution of ischemic injured tissue. Brain Res Bull 35:387-392

8. Demers P, Elkouri S, Martineau R et al (2000) Outcome with high blood lactate levels during cardiopulmonary bypass in adult cardiac operation. Ann Thorac Surg 70:2082-2086

9. De Sommer F (2007) What is optimal flow and how to validate this. J Extra Corpor Technol 39:278-280

10. Dewhurst AT, Moore SJ, Liban JB (2002) Pharmacological agents as cerebral protectants during deep hypothermic circulatory arrest in adult thoracic aortic surgery. A survey of current practice. Anaesthesia 57:1016-1021 
11. Drummond JC (2000) Monitoring depth of anesthesia. Anesthesiology 93:876-882

12. Dullenkopf A, Baulig W, Weiss M et al (2007) Cerebral near-infrared spectroscopy in adult patients after cardiac surgery is not useful for monitoring absolute values but may reflect trends in venous oxygenation under clinical conditions. J Cardiothorac Vasc Anesth 21(4):535-539

13. Edmonds HL, Griffiths L, Laken J van der et al (1992) Quantitative electroencephalographic monitoring during myocardial revascularization predicts postoperative disorientation and improves outcome. J Thorac Cardiovasc Surg 103:555-563

14. Edmonds HL (2001) Advances in neuromonitoring for cardiothoracic and vascular surgery. J Cardiothorac Vasc Anesth 15:241-250

15. Edmonds HL (2004) Monitoring the nervous system during cardiac and vascular surgery. Semin Cardiothorac Vasc Anesth 2:59-60

16. Ellenberger C, Diaper J, Licker M (2007) Bispectral index and detection of acute brain injury during cardiac surgery. Eur J Anaesthesiol 24:803-816

17. Engelhard K, Werner C, Eberspacher E et al (2004) Sevoflurane and propofol influence the expression of apoptosis-regulating proteins after cerebral ischaemia and reperfusion in rats. Eur J Anaesthesiol 21:530-537

18. Engelhard K, Winkelheide U, Werner C et al (2007) Sevoflurane affects neurogenesis after forebrain ischemia in rats. Anesth Analg 104:898-903

19. Fukuda S, Warner DS (2007) Cerebral protection. $\mathrm{Br}$ J Anaesth 99:10-17

20. Grigore AM, Grocott HP, Mathew JP et al (2002) Rewarming rate and increased peak temperature alter neurocognitive outcome after cardiac surgery. Anesth Analg 94:4-10

21. Grocott HP (2006) Perioperative temperature and cardiac surgery. J Extra Corpor Technol 38:77-80

22. Grocott HP, Yoshitani K (2007) Neuroprotection during cardiac surgery. J Anesth 21:367-377

23. Grote CL, Shanahan PT, Salmon P et al (1992) Cognitive outcome after cardiac operations. JThorac Cardiovasc Surg 5:1405-1409

24. Guarracino F (2008) Cerebral monitoring during cardiovascular surgery. Curr Opin Anaesth 21:5054

25. Guerrieri Wolf L, Choudhary BP, Abu-Omar Y et al (2008) Solid and gaseous cerebral microembolization after biologic and mechanical aortic valve replacement: investigation with multirange and multifrequency transcranial Doppler ultrasound. J Thorac Cardiovasc Surg 3:512-520

26. Gugino LD, Agilo LS, Yli-Hankala A (2004) Monitoring the electroencephalogramm during bypass procedures. Semin Cardiothorac Vasc Anesth 8:6183

27. Hans SS, Jareunpoon O (2007) Prospective evaluation of electroencephalography, carotid artery stump pressure, and neurologic changes during 314 consecutive carotid endarterectomies performed in awake patients. J Vasc Surg 3:511-515

28. Head BP, Patel P (2007) Anesthetics and brain protection. Curr Opin Anaesthesiol 20:395-399

29. Himmelseher S, Pfenninger E (2000) Neuroprotektion in der Neuroanästhesie. Die gegenwärtige Praxis in Deutschland. Anaesthesist 49:412-419

30. Hoffmann GM (2006) Neurologic monitoring on cardiopulmonary bypass: what are we obligate to do? Ann Thorac Surg 6:S2373-S2380

31. Hogue CW, Lillie R, Hershey T (2003) Gender influence on cognitive function after cardiac operation Ann Thorac Surg 76:1119-1125
32. Iglesias I, Murkin JM, Bainbridge D et al (2003) Monitoring cerebral oxygen saturation significantly decreases postoperative length of stay: a prospec tive randomized blinded study. Heart Surg Forum 6:204

33. Kadoi Y (2007) Pharmacological neuroprotection during cardiac surgery. Asian Cardiovasc Thorac Ann 15:167-177

34. Kawaguchi M, Furuya H, Patel PM (2005) Neuroprotective effects of anesthetic agents. J Anesth 19:150-156

35. Logemann F, Gras C, Mehler D et al (2001) Bilateral monitoring of cerebral saturation in aortic arch surgery. Anasthesiol Intensivmed Notfallmed Schmerzther 36:388-392

36. Mackensen GB, Ti LK, Phillips-Bute BG et al (2003) Cerebral embolisation during cardiac surgery: impact of aortic atheroma burden. Br J Anaesth 91:656-661

37. Mangano DT (1994) Multicenter outcome research. J Cardiothorac Vasc Anesth 8:10-12

38. Maxwell WL, Watson A, Queen R et al (2005) Slow, medium, or fast re-warming following posttraumatic hypothermia therapy? An ultrastructural perspective. J Neurotrauma 22:873-884

39. Mourisse J, Booij L (2003) Bispectral index detects period of cerebral hypoperfusion during cardiopulmonary bypass. J Cardiothorac Vasc Anesth 17:76-78

40. Murkin JM (2001) Stroke and the elderly: aortic atheroma and CNS outcome after cardiac surgery. Cardiovasc Rev Rep 22:397-398

41. Murkin JM, Adams S, Schaefer B et al (2004) Monitoring cerebral oxygen saturation significantly decreases stroke rate in CABG patients: a randomized blinded study. Heart Surg Forum 7:515

42. Murkin JM (2004) Perioperative multimodality neuromonitoring: an overview. Semin Cardiothorac Vasc Anesth 2: 167-171

43. Murkin JM, Adams S, Novick RJ et al (2007) Monitoring brain oxygen saturation during coronary bypass surgery: a randomized prospective study. Anesth Analg 104:51-58

44. Nabavi DG, Stockmann J, Schmid C et al (2003) Doppler microembolic load predicts risk of thromboembolic complications in Novacor patients. J Cardiothorac Surg 126:1160-1167

45. Newman MF, Mathew JP, Grocott HP et al (2006) Central nervous system injury associated with cardiac surgery. Lancet 368:694-703

46. Nussmeier NA, Arlund C, Slogoff S (1986) Neuropsychiatric complications after cardiopulmonary bypass: cerebral protection by a barbiturate. Anesthesiology $64: 165-170$

47. Nussmeier N (2005) Management of temperature during and after cardiac surgery. Tex Heart Inst J 32:472-476

48. Nussmeier NA, Cheng W, Marino M et al (2006) Temperature during cardiopulmonary bypass: the discrepancies between monitored sites. Anesth Analg 103:1373-1379

49. Ranucci M, Romitti F, Isgro G et al (2005) Oxygen delivery during cardiopulmonary bypass and acute renal failure after coronary operations. Ann Thorac Surg 80:2213-2220

50. Ranucci M, Isgro G, Romitti F et al (2006) Anaerobic metabolism during cardiopulmonary bypass: predictive value of carbon dioxide derived parameters. Ann Thorac Surg 81:2189-2195

51. Ranucci M, de Toffol B, Isgro G et al (2006) Hyperlactatemia during cardiopulmonary bypass: determinants and impact on postoperative outcome. Crit Care 10:R167
52. Rodriguez RA (2004) Human auditory evoked potentials in the assessment of brain function during major cardiovascular surgery. Semin Cardiothorac Vasc Anesth 8:85-99

53. Saidi N, Murkin JM (2005) Applied neuromonitoring in cardiac surgery: patient specific management. Semin Cardiothorac Vasc Anesth 1:17-23

54. Schirmer U (2007) Hypothermie in der Herzchirurgie. Anaesthesist 56:930-935

55. Stecker MM, Cheung AT, Pochettino A et al (2001) Deep hypothermic circulatory arrest: effects of cooling on electroencephalogram and evoked potentials. Ann Thorac Surg 71:14-28

56. Sundt TM Jr, Sharbrough FW, Piepgras DG et al (1981) Correlation of cerebral blood flow and electroencephalographic changes during carotid endarterectomy: with results of surgery and hemodynamics of cerebral ischemia. Mayo Clin Proc 56:533-543

57. Villacorta J, Kerbaul F, Collart F et al (2005) Perioperative cerebral ischaemia in cardiac surgery and BIS. Anaesth Intensive Care 4:514-517

58. Williams GD, Ramamoorthy C (2007) Brain monitoring and protection during pediatric cardiac surgery. Semin Cardiothorac Vasc Anesth 11:23-33

59. Zaidan JR, Klochany A, Martin WM et al (1991) Effect of thiopental on neurologic outcome following coronary artery bypass grafting. Anesthesiology 74:406-411

60. Yao FS, Cheng CC, Ho CY et al (2004) Cerebral oxygen desaturation is associated with early postoperative neuropsychological dysfunction in patients undergoing cardiac surgery. J Cardiothorac Vasc Anesth 5:552-558 